\title{
La arquitectura económica y financiera de la UEM y los efectos de la crisis*
}

\author{
Antonia Calvo Hornero \\ Catedrática de Economía Aplicada
}

\begin{abstract}
Sumario: I. Introducción. - II. La crisis financiera internacional como escenario previo. 1. El Plan coordinado de la UE. - III. La crisis en la UE y en el Eurosistema: la crisis de deuda soberana. 1. La crisis de deuda soberana.-IV. La arquitectura monetaria y financiera de la UEM: La crisis de gobernanza del Eurosistema. 1. La gobernanza monetaria. 2. La gobernanza económica. 3. La gobernanza fiscal. 4. La Gobernanza de la Política Estructural. 5. La gobernanza internacional. - V. Las modificaciones en la gobernanza económica y las medidas financieras: La nueva arquitectura económica y financiera de la UEM. 1. Las medidas monetarias y financieras: el MEDE. 2. Supervisión y sistema bancario: la ABE y la Unión Bancaria.-VI. Conclusión. - VII. Bibliografía.
\end{abstract}

Resumen: La crisis económica y financiera internacional y sus consecuencias en el Eurosistema con la crisis de deuda soberana han terminado afectando a las reglas de funcionamiento en las que estaba basada la UEM y a su estructura institucional habiéndose puesto de manifiesto los fallos que existen en la gobernanza de la UE y en el Eurosistema. La crisis ha acabado mostrando no solo las vulnerabilidades del área de la moneda única, sino también la falta de mecanismos de regulación y supervisión de los desequilibrios macroeconómicos y de las finanzas públicas de los Estados miembros de la Eurozona y la incapacidad para afrontar las consecuencias de un impacto exógeno para el área monetaria como ha sido la crisis financiera internacional. En este artículo se analizan los fundamentos en los que estaba basada el área monetaria y se exponen las principales modificaciones que se han llevado a cabo.

Palabras clave: Unión Económica y Monetaria (UEM), crisis financiera, gobernanza, Eurosistema, deuda soberana.

Abstract: The international financial and economic crisis and its impact on the Eurosystem with the subsequent sovereign debt crisis have affected the operating rules of the EMU and its institutional structure. Both events have highlighted the failures in the governance of the EU and the Eurosystem. They show not only the vulnerabilities of the single currency area but also the lack of adequate regu-

* Recibido el 29 de mayo de 2013, aceptado el 24 de junio de 2013. 
lation and supervision mechanisms of macroeconomic imbalances and public finances of the Member States in the Eurozone and the inability to cope with the consequences of an exogenous shock hitting the currency area as has been the international financial crisis. This article discusses the foundations of the monetary area and the main changes that have been introduced in its rules and procedures.

Keywords: Economic and Monetary Union (EMU), financial crisis, governance, Eurosystem, sovereign debt.

\section{Introducción}

La crisis económica y financiera internacional y sus consecuencias en la Unión Europea con la crisis de deuda soberana han afectado a los principios en los que estaba basada la arquitectura monetaria y financiera de la Unión Económica y Monetaria habiéndose puesto de manifiesto los fallos que existen en la gobernanza de la UE y en el Eurosistema. La crisis ha mostrado lo vulnerable que era la estructura institucional en la que estaba basada el área de la moneda única, la falta de mecanismos de regulación y supervisión de los desequilibrios macroeconómicos y de las finanzas públicas de los Estados miembros de la Eurozona y la incapacidad para afrontar las consecuencias de un impacto exógeno para el área monetaria como ha sido la crisis financiera internacional. La acumulación de todas estas vulnerabilidades y la lentitud en la toma de decisiones a nivel institucional han contribuido activamente al escenario de confusión existente en la UE a partir de mayo de 2010 y al contagio de la crisis a los países del sur de la UE.

A raíz de la crisis de deuda soberana se han llevado a cabo propuestas para evitar que políticas económicas y presupuestarias inadecuadas tomadas por países miembros de la Eurozona, afecten al resto de los Estados miembros del área y puedan poner en riesgo la estabilidad de la moneda única y la viabilidad de la UEM. Así, se ha establecido un nuevo marco de vigilancia macroeconómica para identificar y corregir los desequilibrios macroeconómicos y fiscales. Se han activado dispositivos de seguimiento de estos programas para la corrección temprana de los desequilibrios fiscales y macroeconómicos y se han articulado un conjunto de mecanismos de gestión de las crisis y de apoyo financiero, tanto para los Estados miembros de la UEM (el Mecanismo Europeo de Estabilización Financiera, EFSM y el Mecanismo Europeo de Estabilidad, MEDE), como para el resto de los países de la UE (Facilidad Europea de Estabilidad Financiera, EFSF) y se han sentado las bases para mejorar la cohesión económica en la UE. 
En este artículo se analizan los principales cambios que ha experimentado el Eurosistema como consecuencia de la crisis financiera global y de la crisis de deuda soberana, en función de la arquitectura económica y financiera existente con anterioridad a la crisis y las medidas que se han ido tomado desde que la crisis de deuda soberana puso en evidencia los fallos institucionales y la falta de mecanismos existentes para afrontarlo. Se analiza en primer lugar el escenario internacional provocado por la crisis financiera internacional, en segundo lugar la repercusión económica y financiera en la UE y en el Eurosistema y finalmente las medidas adoptadas para hacer de la UEM un sistema viable a largo plazo.

\section{La crisis financiera internacional como escenario previo}

La crisis financiera internacional se inició en agosto de 2007 y tuvo su origen en el mercado de las hipotecas subprime de Estados Unidos extendiéndose con rapidez por todo el sistema financiero, convirtiéndose éste en un sistema muy frágil a escala global. La respuesta inicial a la crisis fue una intervención masiva y coordinada de los bancos centrales desde el 9 de agosto de 2007, intervenciones que se fueron repitiendo posteriormente con el objetivo de facilitar un desapalancamiento ordenado del sistema, impedir un credit crunch y garantizar la supervivencia de las entidades financieras. La crisis avanza un paso más el 15 de septiembre de 2008 cuando Lehman Brothers Holdings Inc. se declara en quiebra, arrastrando a los otros tres grandes bancos de inversión americanos, con la única opción de ofrecerse en venta o convertirse en instituciones de depósito. Este acontecimiento extiende el riesgo de quiebra a otras instituciones financieras, afecta a las contrapartidas financieras y reduce la liquidez en los mercados de derivados. Al colapsar el conglomerado de seguros AIG, crece la preocupación por los seguros y la posibilidad de rescate por el Sector Publico. Las retiradas masivas de fondos del mercado monetario y algunos cierres de entidades financieras, acaban poniendo en entredicho la viabilidad de las instituciones financieras con necesidad de financiación y se produce el drenaje agudo de liquidez o credit crunch. Tras el desconcierto inicial se reúne el G-20 y el Eurogrupo tratando de lanzar mensajes de tranquilidad para garantizar la supervivencia de las entidades financieras, proponiendo como solución planes de rescate del sector financiero. El primero que lo propone es Estados Unidos el 3 de octubre de 2008 y la UE adopta unos días más tarde el 7 de octubre un compromiso político y el día 13 un plan coordinado. 


\section{El Plan coordinado de la UE}

El plan coordinado de la UE que se inicia el 13 de octubre de 2008, parte del compromiso de los Estados miembros de apoyar al sistema financiero. En el fondo era un plan modelado a iniciativa del Reino Unido, donde Alemania se comprometía a emitir $500 \mathrm{~mm}$ de euros de los cuales hasta $400 \mathrm{~mm}$ eran en garantías de crédito para préstamos interbancarios y un fondo de $100 \mathrm{~mm}$ de euros para inyectar capital a instituciones financieras y adquirir activos no líquidos. Se consideraba la mayor intervención del Estado en tiempos de paz en la economía del país (en el fondo tomaba elementos del US Troubled Asset Relief Programme) y de hecho el ministro de Hacienda alemán advirtió a los bancos de la posibilidad de interferir en sus decisiones, entre las que se contaba la limitación del salario de los ejecutivos a 500 mil euros anuales, la prohibición sobre los bonus y el pagos de dividendos, además de la obligación de prestar a las pymes. En Francia, se habilitaron $360 \mathrm{~mm}$ de euros en garantías de crédito y nuevo capital para los bancos que lo necesitasen (hasta $40 \mathrm{~mm}$ de euros). En el Reino Unido, se habilitaron $375 \mathrm{~mm}$ de euros para tres de los bancos más importantes del país como parte de su anunciado plan de rescate. En Holanda, España, Austria, Italia, Portugal y Noruega en conjunto, se comprometieron a proporcionar más de $500 \mathrm{~mm}$ de euros en garantías y capital. La Reserva Federal americana puso a disposición cantidades ilimitadas de fondos en dólares disponibles offshore, para ser distribuido por el BCE, el Banco de Inglaterra y el Banco Nacional Suizo en el caso de que se produjese una fuerte demanda de dólares en Europa.

Los planes de rescate financiero tuvieron un efecto no deseado sobre la economía real en la UE, provocando el desencadenamiento de un círculo vicioso de la economía debido a la restricción del crédito, la caída de la demanda de consumo (no registrado en décadas), un fuerte descenso en las ventas al por menor, la reducción de los proyectos de inversión de las empresas que previsoramente deciden arrinconar los proyectos y optar por la liquidez ante la incertidumbre. Este círculo vicioso de la economía se traduce con crudeza en una fuerte reducción del empleo. Un año antes de estallar la crisis de deuda soberana del Eurosistema, en 2008, las recomendaciones de la Comisión y del ECOFIN se encaminaban a la necesaria profundización de la vigilancia macroeconómica para volver a una posición fiscal sostenible, ignorando los riesgos del sector bancario, la exposición al sector inmobiliario en algunos miembros de la Eurozona y la desorganización y la falta de acuerdo, como consecuencia de un inadecuado sistema de gobernanza, que se produciría en la toma de decisiones para afrontar en principio el problema griego que se desencadenaría a partir del Consejo Europeo del mes de marzo de 2010 y especialmente en el Consejo Europeo del mes de mayo. 


\section{La crisis en la UE y en el Eurosistema: la crisis de deuda soberana}

La crisis financiera internacional afectó a todos los países de la UE desde 2007 a 2009, pero a partir de mayo de 2010 la crisis se centra en los países de la Eurozona desencadenándose la crisis de deuda soberana. En la primera parte de la crisis financiera internacional, de 2007 a 2008, lo que considero corresponde a la crisis financiera global, la respuesta internacional vino de los Bancos Centrales con intervenciones masivas y coordinadas desde el 9 de agosto de 2007. En esta primera parte de la crisis se producen en la UE efectos institucionales y efectos económicos y financieros. Desde el punto de vista institucional, las consecuencias sobre la UE estaban relacionadas esencialmente con los objetivos que tenía en aquellos momentos la UE, relacionados con la Política de Competencia y el Pacto de Estabilidad y Crecimiento (PEC) y los propios objetivos de la Presidencia del Semestre de la UE (que ostentaba Francia). Al proponerse las Ayudas del Estado para proteger al sistema financiero, se afectaron los objetivos de la Política Competencia de la UE. Dichas ayudas eran contrarias a las normas que rigen una competencia leal en la UE, pero debido a la urgencia de la situación y para agilizar los planes de emergencia se permitieron las ayudas de forma temporal y con condiciones. Las reglas del Pacto de Estabilidad y Crecimiento (PEC) también se vieron afectadas desde el punto de vista institucional al haberse constituido la crisis como un auténtico test de credibilidad para el PEC. La política monetaria y presupuestaria se esperaba que contribuyesen a sostener la demanda y no afectasen al deterioro de las finanzas públicas de los Estados miembros.

\section{La crisis de deuda soberana}

A partir de finales de 2009 se pone de manifiesto una nueva fase de la crisis europea con la crisis de deuda soberana que comenzó en Grecia, provocando unos graves problemas institucionales en la UEM que se manifiestan claramente en el Consejo del mes de mayo de 2010. Los graves desequilibrios macroeconómicos y fiscales en algunos países periféricos llevaron a que los mercados financieros cuestionasen la sostenibilidad de la deuda pública, surgiendo claros riesgos de contagio no solo para los países periféricos afectados también para el resto de la Eurozona y para la moneda única. Para hacer frente a la situación, en el Consejo Europeo del mes de mayo se adoptaron medidas para auxiliar a los países miembros de la UE y del Eurosistema más afectados por la crisis (se creó la Facilidad Europea de Estabilidad Financiera y el Mecanismo Europeo de Estabilización Financiera) y se inició una reforma de la gobernanza económica del Eurosistema. 


\section{La arquitectura monetaria y financiera de la UEM: la crisis de gobernanza del Eurosistema}

Inicialmente, el establecimiento de la UEM tuvo un indudable impulso político pero se sabía que estaba carente de una estructura institucional suficiente cuyos defectos se habían puesto de manifiesto en la redacción del Tratado de Maastricht. Se consideraba (más por el deseo que por razones técnicas) que la moneda única iba a responder a una serie de objetivos económicos, como era la estabilidad macroeconómica, el crecimiento del empleo y una mejora de la cohesión y de la convergencia de los Estados miembros. También se sabía que la creación de la moneda única no estaba exenta de posibles riesgos asociados debido especialmente a una política fiscal descentralizada en las decisiones de los gobiernos nacionales, a diferencia de los Estados federales, donde las decisiones monetarias y fiscales las asume el gobierno federal actuando como un estabilizador automático con transferencias fiscales para los estados que lo necesitan, mientras que en la UE se carece de un presupuesto centralizado para afrontar los problemas comunes y las perturbaciones externas con efectos asimétricos. Si a esto añadimos una ineficaz supervisión de las políticas macroeconómicas y del sistema financiero y un sistema de gobernanza y de órganos de decisión insuficientes para afrontar una crisis sistémica como la actual, podemos comprender el alcance y profundidad de la crisis de deuda soberana y sus efectos en el Eurosistema.

El sistema de gobernanza de la UE se estableció en el Tratado de Maastricht (T de M) para asegurar el funcionamiento de la Unión monetaria, habiéndose estructurado alrededor de cuatro grandes pilares: 1. la gobernanza monetaria, 2. la gobernanza fiscal, 3. la gobernanza de la política estructural, y 4. la gobernanza internacional. De los cuatro grandes pilares de la gobernanza europea, la gobernanza monetaria y la gobernanza fiscal son las más relevantes para el funcionamiento de la moneda única.

\section{La gobernanza monetaria}

La gobernanza monetaria está organizada alrededor del BCE como Banco Central independiente. Su objetivo principal es la estabilidad de precios y solo en segundo lugar y siempre que no se vea afectado ese objetivo primordial deberá apoyar las políticas económicas generales de la UE. Aunque la gobernanza monetaria corresponde de forma exclusiva al BCE existen otros órganos comunitarios que colaboran en esta función, como es el ECOFIN o Consejo de Ministros de Economía y Finanzas de la UE, porque un conjunto de políticas comunes se deciden por el Consejo Europeo. 
Si bien el Tratado de Maastricht otorga casi exclusivamente un mandato de política monetaria al $\mathrm{BCE}$, se dejaron abiertas algunas cuestiones básicas estrechamente relacionadas con la política monetaria y que en la crisis de deuda soberana han emergido como asuntos a resolver para el futuro funcionamiento de la Eurozona. Entre estas cuestiones que quedaron pendiente en el Tratado esta la supervisión bancaria, en la que el BCE tenía un papel muy limitado y la función de prestamista en última instancia, como responsabilidad que tienen los Bancos Centrales de proporcionar liquidez a las instituciones financieras individuales, ante el riesgo de crisis bancaria. Con la crisis financiera internacional y especialmente con la crisis de deuda soberana, el $\mathrm{BCE}$ ha tenido que arbitrar medidas no convencionales, inéditas hasta ese momento y ha estado actuando como proveedor de liquidez para las entidades bancarias del Eurosistema que lo han necesitado. La mayoría de estas nuevas medidas de carácter cuantitativo forman parte del panel de instrumentos que disponen los bancos centrales, porque se consideran medidas de provisión de liquidez. Sin embargo, el volumen y alcance de estas medidas han sobrepasado con creces los niveles convencionales utilizados por los bancos centrales hasta la actual crisis.

Con la crisis de deuda soberana, el BCE ha reducido los tipos de interés de referencia, ha reducido los requerimientos de reservas, ha intervenido en el funcionamiento de los mercados de intermediación a través del Programa de Mercado de Valores (Securities Market Program) y ha proporcionado apoyo de liquidez excepcional para los bancos a través de un nuevo programa de refinanciación colateralizada de tres años con un amplio criterio de colateral elegible. Aparte de las intervenciones puntuales del su Presidente desde julio de 2012 (apoyando directamente al euro e indirectamente a la crítica situación en los mercados de España e Italia), el Banco dio un paso decisivo en apoyo de la UEM al asumir en 2012 la adquisición ilimitada de deuda soberana en el mercado secundario del país que solicite previamente la ayuda financiera al Eurogrupo. Con ello, lo que se ha conseguido sobre todo es asegurar que la UEM sea un proceso irreversible, se puedan recuperar los canales de transmisión de la política monetaria y se puedan corregir las diferencias de las condiciones de financiación del ciclo económico de los Estados miembros.

\section{La gobernanza económica}

La gobernanza económica está basada en la coordinación de las políticas económicas en la UE. Esta se realiza en el marco de las Directrices de Empleo, en las Orientaciones Generales de Política Económica y en la Estrategia Europa 2020, donde se establecen recomendaciones para las au- 
toridades nacionales. Pero se carecen de mecanismos de vigilancia y seguimiento del cumplimiento de dichas recomendaciones y como no son vinculantes, pueden ser ignoradas por los Estados miembros. Esto hace que la gobernanza económica establecida haya mostrado su incapacidad para prevenir los efectos de la crisis y sus consecuencias para el funcionamiento del área monetaria.

Una serie de factores se han dado cita para favorecer la evolución que ha experimentado la Eurozona desde que se desencadenó la crisis de deuda soberana. En primer lugar, unas expectativas excesivamente optimistas de los gobiernos nacionales y la propia Comisión Europea, infravalorando la ausencia de políticas de salarios y de rentas que preservasen la competitividad de la Unión Monetaria. En segundo lugar, unas políticas fiscales, de supervisión y de regulación insuficientes y con unas rigideces estructurales que han abonado la falta de competitividad de gran parte de los Estados miembros. Bien es verdad que con las intervenciones de los gobiernos para hacer frente a la crisis financiera internacional y la acumulación de riesgos por la estabilización del sector bancario, se acabó afectando a los países de la Eurozona que tenían una alta exposición de su sector financiero privado y de forma especial a las finanzas públicas de esos países. La integración de los mercados financieros del área del euro hizo el resto, extendiendo la crisis a otros países del área y poniendo en riesgo la viabilidad de la Eurozona.

\section{La gobernanza fiscal}

De los cuatro tipos de gobernanza que afectan a los países del área del euro, la gobernanza fiscal tiene una importancia crucial para la estabilidad y credibilidad de la moneda única, porque con una unión monetaria la disciplina presupuestaria es indispensable dada la independencia fiscal que tienen los gobiernos participantes en el Eurosistema. Si bien la UE tiene una larga tradición de déficit público que está relacionada en gran parte con el papel que se ha otorgado al Sector Publico en la economía y a la consideración de su eficiencia, al introducirse el criterio de las finanzas públicas en el $\mathrm{T}$ de $\mathrm{M}$ se optó por la precaución y la prevención para asegurar una disciplina fiscal en la Eurozona, con lo que este espíritu de respeto a las decisiones nacionales que se trasladó al Tratado en el criterio de las finanzas públicas se le dio una consideración poco precisa.

Para salvar las evidentes lagunas del Tratado y salvaguardar la estabilidad de la Eurozona se estableció el Pacto de Estabilidad y Crecimiento en el Consejo Europeo de Ámsterdam, de junio de 1997 (fue reformado en 2005 y esta reforma lo debilito seriamente). El PEC carecía de instrumentos 
de aplicación automática en caso de incumplimiento y los previstos tenían un amplio margen de discrecionalidad. La falta de cumplimiento de las reglas del PEC (según el ECOFIN, a principios de 2008, solo 8 de los 17 Estados miembros de la Eurozona se ajustaban a sus objetivos presupuestarios a medio plazo en 2007), más los efectos de la crisis de deuda soberana con los planes de apoyo al sistema bancario y a la demanda interior, colocaron a los Estados miembros del área monetaria en una situación de clara vulnerabilidad, especialmente a los países periféricos.

Además de que nunca se aplicaron las sanciones económicas previstas para corregir un déficit persistente y excesivo, los datos proporcionados por algunos Estados miembros no eran fiables y Eurostat carecía de facultades para obtener las estadísticas nacionales adecuadas y para auditar a los responsables estadísticos de los Estados miembros. El segundo elemento de gobernanza fiscal que aparecía en el T de M, era la cláusula de No Bail Out o cláusula de no corresponsabilidad financiera. Esta ha quedado totalmente invalidada en la crisis de deuda soberana con la decisión de acudir en ayuda de los países con amenaza de quiebra financiera, decidido en el Consejo Europeo del mes de mayo de 2010 y en las siguientes actuaciones del BCE y de la Comisión Europea haciendo necesaria una reforma de la gobernanza económica de la Eurozona.

La aplicación del PEC está basada en dos elementos fundamentales que si bien se han aplicado no se ha hecho con total eficacia, como son: 1. el instrumento preventivo, y 2. el instrumento correctivo. El instrumento preventivo está sustentado en la supervisión multilateral de las situaciones presupuestarias. Consiste en la presentación anual por los Estados miembros de la zona del euro de los denominados Programas de Estabilidad donde señalan los objetivos a cumplir en las finanzas públicas. Los países que aún no participan en la Eurozona también deben presentar programas pero estos se denominan Programas de Convergencia. Ambos programas, tanto los de los países de la moneda única, como los del resto de la UE sirven de base de forma preventiva para que la Comisión lleve a cabo la supervisión multilateral.

Una vez analizados los programas, la Comisión sugiere las recomendaciones pertinentes y como hemos visto con la crisis, no se siguen por todos los países. El instrumento correctivo está apoyado en el procedimiento de déficit excesivo que tiene como principal objetivo impulsar su corrección. Todos estos mecanismos de gobernanza fiscal han sido obviados por la mayoría de los Estados miembros del Eurosistema y es donde se han propuesto las reformas más importantes, con previsión de medidas de corrección en función de la identificación por la Comisión de desequilibrios macroeconómicos o fiscales severos por pérdidas de competitividad que puedan suponer un riesgo para la Eurozona. 


\section{La Gobernanza de la Política Estructural}

La necesidad de impulsar las reformas estructurales está ampliamente aceptada por los Estados miembros de la UE. Incluso el $\mathrm{T}$ de $\mathrm{M}$ en el art. 99.1 requiere a los Estados miembros «considerar sus políticas económicas como un asunto de preocupación común...». Las reformas estructurales son con el ajuste interior, las condiciones necesarias que se deben cumplir en una unión monetaria para que esta pueda funcionar con eficiencia.

\section{La gobernanza internacional}

La gobernanza internacional afecta a la política de tipo de cambio, a la representación internacional y a temas de interés para la UEM. El Tratado contempla la posibilidad de un sistema de tipos de cambio fijos con países fuera de la UE y la formulación de orientaciones generales para la política de tipo de cambio, especialmente referido esto último a los acuerdos informales u otro tipo de acuerdos unilaterales con socios fuera del área del euro. El euro se ha convertido en la segunda moneda internacional detrás del dólar y la primera moneda regional del mundo, dado el peso económico de la Eurozona en la economía internacional y su influencia en la región.

\section{Las modificaciones en la gobernanza económica y las medidas financieras: La nueva arquitectura económica y financiera de la UEM}

Para asegurar la viabilidad de la Eurozona han sido necesarias nuevas medidas en diversos frentes para prevenir y afrontar los posibles desequilibrios macroeconómicos y presupuestarios que puedan aparecer en el futuro. También ha sido necesario apoyar a los países y a los bancos con mayor necesidad de asistencia financiera, papel que ha desempeñado a lo largo de la crisis el BCE, con una política monetaria acomodaticia y la compra (ilimitada y esterilizada) de deuda soberana en el mercado secundario (en vencimientos de 1 a 3 años) a través del programa de Operaciones Monetarias Directas (OMT).

Partiendo de la preocupación por asegurar la viabilidad de la UEM, el Consejo Europeo del mes de marzo de 2010 encomendó al Presidente del Consejo Europeo, Herman Van Rompuy que organizase junto con la Comisión Europea, un grupo de trabajo formado por representantes de los países miembros más el Banco Central Europeo (BCE) y la presidencia rotatoria del Consejo. A este grupo de trabajo conocido por sus siglas GTVR, se le 
encomendó formular propuestas con una triple finalidad: 1. Establecer un mecanismo de gestión de crisis, 2. Reforzar el marco de vigilancia de la UE y 3. Organizar un marco institucional reforzado. El 17 de junio de 2010, el Consejo Europeo decidió aplicar una de las recomendaciones del GTVR sobre gobernanza económica (el Consejo aprobó en octubre el informe presentado por el GTVR y en el mes de diciembre consideró la necesidad de crear un mecanismo permanente gestión de crisis). En concreto la relacionada con el refuerzo de la dimensión ex ante de la coordinación de la política económica, estableciendo a partir del 1 de enero de 2011 lo que se denomina Semestre Europeo (SE).

Con la instauración del Semestre Europeo se pretende coordinar mejor las políticas económica y presupuestaria a escala comunitaria antes de que los gobiernos nacionales adopten decisiones que puedan afectar al resto de los Estados miembros y al equilibrio del sistema. El SE incluye un calendario para la vigilancia tanto de la política macroeconómica como estructural y fiscal. Ese calendario comienza en enero con la publicación del Informe Anual de Crecimiento de la Comisión Europea. En el mes de marzo se celebra la cumbre económica anual del Consejo Europeo donde se señalan las directrices estratégicas que los Estados miembros tendrán que tener en cuenta en sus Programas de Estabilidad y Convergencia (que se remiten en el mes de abril).

En junio de 2010, el Consejo Europeo había aprobado la Estrategia Europa 2020 en sustitución de la Estrategia de Lisboa para el crecimiento y el empleo del año 2000. La Estrategia Europa 2020 tiene como objetivo promover el crecimiento económico sostenible y la creación de elevados niveles de empleo y productividad. Las reformas que se están llevando a cabo en la UE como consecuencia de los problemas planteados por la crisis de deuda soberana se enmarcan en esta estrategia. De esta manera, los Estados miembros dentro del marco de la Estrategia Europa 2020, en sus Programas Nacionales de Reforma deben identificar los obstáculos relacionados con el crecimiento y establecer una estrategia de reformas para fomentar el crecimiento económico sostenible y el empleo. El SE inició su andadura en diciembre de 2011 con el Informe Anual de Crecimiento presentado por la Comisión Europea.

En base a los Programas de Estabilidad y Crecimiento y a los Programas Nacionales de Reforma, el Consejo Europeo debe formular las recomendaciones de política que considere apropiadas para el próximo año antes de que se presenten los presupuestos nacionales en otoño. En este marco de vigilancia se ha previsto un mecanismo de alerta formado por un conjunto de indicadores macroeconómicos, que con el informe de la Comisión sobre la posibilidad de existencia de desequilibrios macroeconómicos en los Estados miembros, pueda servir para conocer con antelación las vulnerabi- 
lidades macroeconómicas y los riesgos potenciales de desequilibrios de los Estados miembros.

Para los desequilibrios externos se utilizan datos de la posición externa como la cuenta corriente, datos de competitividad o costes laborales unitarios y para los desequilibrios internos, precios de la vivienda o valor añadido de la construcción, además de datos relacionados con el endeudamiento interior, tanto del sector público como privado. En la publicación del Informe Anual de Crecimiento de la Comisión al año siguiente, se deben recoger las valoraciones sobre el cumplimiento de las recomendaciones que se le han asignado a los Estados miembros. En el caso de incumplimiento se prevén sanciones que pueden llegar al 0,2\% del PIB del país incumplidor.

Avanzando en las reformas y con el objeto de mejorar la gobernanza, el 29 de septiembre de 2010 se decidió adoptar una serie de medidas legislativas como el denominado Six Pack o conjunto de seis propuestas legislativas (cinco Reglamentos y una Directiva de la Comisión) que entro en vigor en enero de 2012 y desde entonces se lleva a cabo como parte del Semestre Europeo. Esta propuesta se ha completado con el Two Pack (adoptado en el Consejo Europeo del 13 mayo de 2013) o conjunto de regulaciones para mejorar la gobernanza económica de la Eurozona, que incluye una regulación para monitorizar y establecer planes presupuestarios de los Estados miembros del Eurosistema con el fin de prestar una mayor vigilancia de la que está prevista en el procedimiento de déficit excesivo y para aquellos Estados miembros de la Eurozona que están experimentando o están amenazados con serias dificultades financieras.

En 2011 se siguió avanzando en las propuestas con iniciativas relevantes para la reforma de la arquitectura económica de la UE y de la Eurozona. Así, el 21 de junio de 2011 en la Cumbre del Euro se puso de manifiesto la necesidad de incrementar la capacidad de reacción financiera de la UEM ante la crisis y en el mes de septiembre ya se hicieron propuestas en base a una serie de iniciativas enmarcadas en una amplia estrategia para afrontar los aspectos más acuciantes de la crisis de deuda soberana y poder avanzar hacia una mayor integración económica y estabilidad financiera de la Eurozona. En este marco de reformas, se ha establecido el Pacto por el Euro Plus como acuerdo intergubernamental por el que los Estados miembros del Eurosistema y otros de la UE se comprometen a introducir reformas en áreas concretas como el empleo, la competitividad, la estabilidad del sistema financiero y la sostenibilidad de las finanzas públicas. El Pacto fue aprobado por los jefes de Estado o de gobierno de los países del área de la moneda única al que se sumaron también, Bulgaria, Dinamarca, Letonia, Lituania, Polonia y Rumanía y está abierto al resto de los Estados miembros de la Unión. 
Existe un firme compromiso impulsado por Alemania, para la aplicación de medidas adicionales a nivel nacional relacionadas con la adopción a nivel nacional de normas de equilibrio presupuestario en términos estructurales que reflejen el PEC en la legislación nacional, con rango constitucional si es posible o equivalente (Alemania lo tiene incorporado desde 2009 y España incorporó una clausula en el artículo 135 revisado de la Constitución al establecer el principio de estabilidad presupuestaria. La nueva redacción de ese artículo se publicó en el BOE el 27 de septiembre de 2011).

En definitiva, se pretende mantener una supervisión más estricta de la Eurozona donde los países sometidos al procedimiento de déficit excesivo, estarán sometidos al análisis por la Comisión y el Consejo de los proyectos de presupuestos nacionales pudiendo pronunciarse al respecto, antes de que los parlamentos nacionales los adopten, a la vez que la Comisión podrá supervisar la ejecución presupuestaria y si es necesario podrá sugerir modificaciones a lo largo del año, de forma, que si se detecta cualquier desviación de un programa de ajuste, se podrá supervisar y coordinar de una forma más estricta (art. 136 del Tratado Fundamentos de la UE).

\section{Las medidas monetarias y financieras: EL MEDE}

En el Consejo Europeo de mayo de 2010 se decidió crear dos mecanismos de carácter temporal, el Mecanismo Europeo de Estabilización Financiera (MEEF) para los países de la UE y la Facilidad Europea de Estabilización Financiera (FEEF) específica para la Eurozona. La temporalidad de ambos mecanismos se ha resuelto con un mecanismo más definitivo, el MEDE o Mecanismo Europeo de Estabilidad para la resolución de crisis de carácter permanente en los países de la Eurozona. Este mecanismo se activara cuando sea necesario para salvaguardar la estabilidad del Eurosistema. En el Consejo Europeo del 16 y 17 de diciembre de 2010 se acordó introducir una modificación del Tratado para establecerlo. Este mecanismo sustituye a los creados en mayo de 2010 vigentes hasta junio de 2013.

El Mecanismo Europeo de Estabilidad refuerza el nuevo marco de gobernanza económica europea y el país que lo solicite deberá cumplir un programa estricto de ajuste económico y fiscal, a la vez que el FMI y la Comisión, en contacto con el BCE, evaluaran la sostenibilidad de la deuda del país solicitante. Está previsto que tanto el FEEF como el MEDE sean utilizados de forma flexible, lo que significa que pueden existir alternativas de financiación variadas, como la activación de la compra de deuda en el mercado secundario por el MEDE con el BCE como agente y con la condicionalidad exigida.

Como condición previa a la concesión de la ayuda a los países que lo soliciten tiene que haber acuerdo unánime de los países del Eurosistema y 
el país deudor tiene que negociar un plan general de reestructuración con los acreedores, a la vez que todos los bonos de nueva emisión del Estado miembro de la Eurozona deben incluir a partir de junio de 2013 unas cláusulas de acción colectiva normalizadas e idénticas. Con la posible participación del sector privado en caso de insolvencia de un país de la Eurozona, se puede garantizar que los tipos de interés reflejen lo mejor posible los riesgos asociados a las diferentes situaciones presupuestarias de los Estados miembros. El MEDE también podrá recapitalizar directamente a las entidades bancarias con problemas para reducir las tensiones en los mercados de deuda, siempre que previamente se haya establecido el sistema de supervisión única bancaria de la Eurozona.

Uno de los efectos que considero más importante es que el MEDE puede contribuir con sus condiciones estrictas a reducir el riesgo moral de la Eurozona, tan afectado en la crisis de deuda soberana. El riesgo moral o percepción de la existencia de un respaldo financiero ante momentos de crisis, alienta un comportamiento más imprudente por inversionistas y deudores. En mi opinión, en el área monetaria europea este concepto lo podemos extender a la actuación no sólo de inversionistas o instituciones financieras, también se ajustaría al comportamiento irresponsable de algunos gobiernos de la Eurozona que han actuado como free rider esperando que en el caso de crisis de deuda soberana (como ha ocurrido), el resto de los miembros del área acudirían de alguna manera a su rescate, para evitar el colapso del sistema (como ha ocurrido). Esa es la razón por la que considero que el Mecanismo Europeo de Estabilidad puede contribuir con sus condiciones estrictas a reducir el riesgo moral de la Eurozona.

\section{Supervisión y sistema bancario: la ABE y la Unión Bancaria}

La crisis no solo ha mostrado las lagunas existentes en la gobernanza económica y monetaria, también ha puesto de manifiesto la fuerte interconexión entre los sistemas financieros de los Estados miembros de la unión monetaria y la relación entre soberanía y sistema bancario. Por esa razón se ha contemplado la reforma financiera con un amplio planteamiento paneuropeo al proponer establecer: 1. Una Autoridad Bancaria Europea, 2. El fortalecimiento del sistema bancario y 3. La propuesta de una unión bancaria y un fondo de garantía de los depósitos centralizado.

La Autoridad Bancaria Europea (ABE) se estableció por el Reglamento (UE) n. ${ }^{\circ}$ 1093/2010 del Parlamento Europeo y el Consejo de 24 noviembre 2010 por el que se crea una Autoridad Europea de Supervisión o Autoridad Bancaria Europea (derogándose la Decisión 2009/78/CE de la Comisión y modificando la Decisión n. ${ }^{\circ}$ 716/2009/CE. La componen los bancos centra- 
les de la UE y los representantes de supervisores bancarios de los Estados miembros. Su objetivo es salvaguardar la estabilidad financiera y la eficacia del sistema bancario con especial interés por el riesgo sistémico que pueden plantear las entidades financieras. Asesora a la Comisión y facilita la aplicación de las normas comunitarias relacionadas con la supervisión financiera, aunque también puede desarrollar actividades relacionadas con la protección de los consumidores. Entre las recomendaciones que ha llevado a cabo está el requerimiento a los bancos para que se desprendan de activos y poder cumplir el objetivo del 9\% del objetivo del capital, también se les ha solicitado que cubran el déficit sobre todo con medidas de capital y se ha llevado a cabo un requerimiento a los bancos para incrementar las posiciones de capital, incluyendo límites para tratar el riesgo soberano.

El Reglamento por el que se crea la ABE, forma parte de las medidas adoptadas para reformar el Sistema Europeo de Supervisión Financiera (SESF), junto a la creación de otros tres organismos de supervisión financiera, la Autoridad Bancaria de Seguros y Pensiones de Jubilación, la Autoridad Europea de Valores y la Junta Europea de Riesgo Sistémico. Las actividades del Comité Mixto de las Autoridades Europeas de Supervisión y las actividades de las autoridades encargadas de la supervisión o con responsabilidades afines en cada Estado miembro, forman parte también de la supervisión financiera, así como la Directiva denominada Ómnibus (y la adaptación de la legislación a las nuevas estructuras de supervisión con la Directiva Ómnibus II).

Formando parte del conjunto de reformas planteadas a partir de mayo de 2010 (y que aparecen en el informe presentado por el Presidente del Consejo Europeo en junio de 2010: Hacia una genuina Union Economica y Monetaria, donde se identifican cuatro elementos esenciales como base de la UEM: 1. el establecimiento de un marco financiero integrado o Union Bancaria, 2. Un marco presupuestario integrado, 3. Un marco integrado de política económica y 4. La involucración del Parlamento Europeo y de los parlamentos nacionales.

La Unión Bancaria, si se establece, contará con un sistema de supervisión bancaria centralizada en el BCE, compartiendo parte de la función supervisora con las autoridades nacionales en función del tamaño y naturaleza de las entidades. Contará también con un sistema de garantía de depósitos y un mecanismo de resolución de entidades con problemas de permanencia, financiado con las contribuciones de los bancos para romper la interrelación entre riesgo bancario y riesgo soberano, aunque el MEDE puede que tenga que ejercer la función de aportador financiero de última instancia. En parte por la falta de concreción de algunas de las medidas propuestas relacionadas con la unión bancaria y el aparente rechazo por Alemania, hacen que tras la buena acogida inicial por los mercados que aliviaron las tensiones en 
el diferencial de intereses con Alemania, especialmente de España e Italia, ésta acogida se haya traducido en cierta desconfianza de que se pueda lograr en un plazo razonable.

Si la propuesta de la unión bancaria llega a buen puerto y se logra un marco presupuestario integrado, pasada una primera etapa de reforzamiento de la disciplina presupuestaria, se podría en el largo plazo llegar a emisiones conjuntas de deuda pública con responsabilidad compartida e incluso una unión fiscal, con presupuesto centralizado y un Tesoro público europeo. El marco integrado de política económica exigirá que las recomendaciones que están enmarcadas en el Semestre Europeo y en el Pacto por el Euro Plus sean más vinculantes que en la actualidad. Ahondando en las responsabilidades institucionales compartidas y en la necesidad de fortalecer la legitimidad democrática y la transparencia del proceso, se considera conveniente involucrar al Parlamento Europeo y a los parlamentos nacionales.

Otras medidas propuestas guardan relación con el establecimiento del Pacto por el Crecimiento y el Empleo relacionado con las recomendaciones del Semestre Europeo y los objetivos de la Estrategia Europa 2020. A través de este Pacto se podrán movilizar $120 \mathrm{~mm}$ de euros para llevar a cabo inversiones en infraestructuras (a través del aumento de $10 \mathrm{~mm}$ de euros del capital desembolsado del BEI, (que incrementaría de esta manera su capacidad de financiación hasta $60 \mathrm{~mm}$ de euros) además del lanzamiento de la fase piloto con $5 \mathrm{~mm}$ de euros de los «bonos para proyectos». Con esto más otras medidas que se habiliten en el futuro, se pretende apoyar la innovación, el empleo juvenil y las pymes para impulsar el crecimiento en la UE, tan necesario para poder crear empleo.

\section{Conclusión}

Desde el inicio de la crisis de deuda soberana en mayo de 2010 se han ido tomando medidas para paliar los efectos que la crisis financiera internacional ha provocado en los países miembros de la UE y en la UEM. La UE ha puesto en marcha en ese tiempo un conjunto de medidas encaminadas a garantizar unas cuentas públicas saneadas en los Estados miembros, mecanismos de apoyo financiero para los países y para los bancos con problemas y se han puesto las bases para la mejora del sistema de gobernanza con el que se había dotado a la UEM desde sus inicios. La coordinación de las políticas económicas se ha mejorado sustancialmente con la instauración del Semestre Europeo y el Plan Euro Plus y con los dos paquetes de medidas legislativas, que permiten una mayor coordinación económica y presupuestaria a nivel europeo antes de que los Estados miembros adopten las decisiones nacionales. 
Desde el punto de vista comunitario se han activado dispositivos de seguimiento de los programas para la corrección temprana de los desequilibrios fiscales y macroeconómicos y se han articulado un conjunto de mecanismos de gestión de la crisis y de apoyo financiero, tanto para los Estados miembros de la UEM (el Mecanismo Europeo de Estabilización Financiera, MEEF y el Mecanismo Europeo de Estabilidad, MEDE), como para el resto de los países de la UE (Facilidad Europea de Estabilidad Financiera, FEEF).

Se han puesto en marcha importantes reformas para establecer las instituciones necesarias que posibiliten el funcionamiento de una unión monetaria sostenible, al aprobarse un Pacto de Estabilidad Fiscal (que si bien en parte redunda en las obligaciones existentes, en mi opinión puede contribuir a reforzar aún más el compromiso de estabilidad presupuestaria requerido en una unión monetaria, dada la escasa inclinación que hasta ahora han mostrado algunos gobiernos de la Eurozona para cumplir los pactos fiscales acordados), incluido en el nuevo Tratado de Estabilidad, Coordinación y Gobernanza adoptado en enero de 2012 y especialmente con la propuesta en junio de 2012 de establecer una Unión Bancaria. Se ha fortalecido el PEC, se han introducido nuevos mecanismos para prevenir o corregir los desequilibrios macroeconómicos y existe una mayor coordinación de las políticas estructurales.

Además, se han sentado las bases para mejorar la cohesión económica en la UE incluyendo: 1. Un marco financiero integrado a nivel europeo, 2. Mayor corresponsabilidad en el diseño de las políticas económicas, 3. Mayor integración fiscal, y 4. Mejora de la transparencia y de la rendición de cuentas. Pero sobre todo se ha tomado conciencia de que la responsabilidad es conjunta, de que es necesario organizar una estructura institucional más adecuada y con mayor poder coercitivo y se debe llevar a cabo una reforma de la gobernanza económica de la Eurozona, sin afectar al Tratado para no prolongar excesivamente en el tiempo las reformas.

En consecuencia, se ha establecido un nuevo marco de vigilancia macroeconómica para identificar y corregir los desequilibrios macroeconómicos que complementa el proceso de vigilancia macroestructural previsto en la Estrategia Europa 2020. Este nuevo marco de vigilancia está previsto que se aplique en todos los Estados miembros con una vertiente preventiva y otra correctiva (en este caso, con normas de aplicación exclusiva para los países de la Eurozona). En los países más afectados por fuertes tensiones económicas y financieras, como Grecia, Portugal, Irlanda y Chipre. En España con la asistencia para la recapitalización de algunos bancos españoles. Esta ayuda se ha llevado a cabo en primera instancia a través de la Facilidad Europea de Estabilidad Financiera (FEEF) y se ha transmitido al MEDE a partir de junio de 2013, sin que en este traspaso el MEDE haya adquirido el estatus de acreedor preferente. Se han puesto en marcha reformas en los 
mercados de bienes y de factores y en sus sistemas bancarios y se han implementado programas de consolidación fiscal para enderezar las finanzas de estos países.

Pero la crisis no solo ha mostrado las lagunas existentes en la gobernanza económica y monetaria, también ha puesto de manifiesto, la fuerte interconexión entre los sistemas financieros de los Estados miembros de la unión monetaria y la relación entre soberanía y sistema bancario. Por esa razón, se ha contemplado la reforma financiera con un amplio planteamiento paneuropeo con el establecimiento de una Autoridad Bancaria Europea, el fortalecimiento del sistema bancario y la propuesta de un fondo de garantía de los depósitos centralizado.

Con la Unión Bancaria y el establecimiento de un mecanismo único de supervisión bajo la responsabilidad del BCE para corregir la fragmentación de los mercados financieros, se podrá posibilitar la recapitalización directa por el MEDE de los bancos que se encuentren en dificultad. Con todas estas reformas se espera que los mercados recuperen la confianza en la UE y en el funcionamiento del Eurosistema y se alivie la presión que tiene la deuda soberana de los países periféricos del área monetaria común. En definitiva hacer viable la Unión Económica y Monetaria.

\section{Bibliografía}

Bolton, P. and O. Jeanne, «Sovereign default risk and bank fragility in financially integrated economies», IMF Economic Review, 59 (2), 2011 pp. 162-194.

CALvo, A., «La crisis de las hipotecas subprime y el riesgo de credit crunch». Revista de Economia Mundial, Univ. de Huelva, n. ${ }^{\circ}$ 18, 2008.

CALvo, A., «Crisis financiera global y arquitectura institucional internacional», en Lecciones de la crisis, Revista del Instituto de Estudios Económicos, n. ${ }^{\circ} 1 /$ 2010.

CAlvo, A., «La toma de decisiones en la UE y en la UEM», en La crisis de la Unión Monetaria Europea, n. ${ }^{\circ}$ 133, Economistas, Colegio de Economistas de Madrid, Madrid 2012.

BCE (nota del presidente del BCE), «Reforzamiento de la gobernanza económica en la zona del euro», 15 de junio de 2010, (http://www.ecb.europa.eu/pub/pdf/ other/reinforcingeconomicgovernance

BDE (informe trimestral de la economía española), Boletín Económico, julio-agosto de 2012.

De Grauwe, P., A Self-inflicted Crisis, Euro Intelligence, 29 de mayo de 2012.

DE GRAuwe, P., In search of Symmetry in the Eurozone, CEPS Policy Brief, no. 268 May 2012.

ECB, Changes in bank financing patterns, April 2012a.

ECB, Financial Integration in Europe, April 2012b.

IMF, World Economic Outlook, October 2012. 